Erschienen in: Eichinger, Ludwig M./Kallmeyer, Werner (Hrsg.): Standardvariation.

Wie viel Variation verträgt die deutsche Sprache?. - Berlin, New

York: de Gruyter, 2005. S. 324-333. (Institut für Deutsche Sprache. Jahrbuch 2004),

https://doi.org/10.1515/9783110193985.324

\author{
STEPHEN BARBOUR
}

\title{
Standardvariation im Deutschen und im Englischen: Auswirkungen auf die Kommunikation zwischen Sprechern beider Sprachen*
}

\begin{abstract}
Da das britische Englisch im Wesentlichen nur Gebrauchsnormen kennt, und keine Sollnormen, bedeutet Standardvariation im Deutschen für Englischsprachige kein grundsätzliches Hindernis beim Erlernen der Sprache. Umgekehrt werden deutschsprachige Lerner des Englischen immer wieder durch das Fehlen von Sollnormen im Englischen irritiert.

Neuerdings entstehen aber Schwierigkeiten auf beiden Seiten durch Änderungen in der Lexik des Deutschen, die als Lockerungen der Norm aufgefasst werden könnten. Es handelt sich hier um die Verwendung von Anglizismen in deutschen Texten. Vermutlich, weil diese oft als nicht normgerecht betrachtet werden, werden sie nur zögernd in Nachschlagewerke aufgenommen, was Lernern der Sprache Schwierigkeiten bereitet. Da es sich hier angeblich um „englische Wörter" handelt, könnte der Eindruck entstehen, sie seien für Englischsprachige unproblematisch. Die deutschen Anglizismen sind aber keine „englischen Wörter", sie weichen im Gebrauch und in der Bedeutung oft erheblich von ihren englischen Vorbildern ab.

Auf der anderen Seite neigen deutschsprachige Lerner des Englischen oft dazu, vermutlich irregeführt durch die übliche Bezeichnung „englische Wörter", deutsche Anglizismen nach deutschen Gebrauchs- und Bedeutungsmustern in ihren englischen Äußerungen zu verwenden, was unter Umständen zu erheblichen Missverständnissen führen kann. Es könnte sogar argumentiert werden, es entstehe durch diese und andere Prozesse eine neue Standardvariante des Englischen, ein ,deutsches Englisch“

In diesem Referat werde ich empfehlen, auf die irreführende Bezeichnung „englische Wörter" für deutsche Anglizismen zu verzichten. Ich werde auch dafür plädieren, die Standardvariation so früh wie möglich im Sprachunterricht zu berücksichtigen, da sie für das Verständnis sowohl gesprochener als auch geschriebener Sprache von großer Bedeutung sein kann.
\end{abstract}

* Für wichtige Hinweise bin ich vielen Kollegen zu Dank verpflichtet, insbesondere Alan Cornell (Plymouth), Alan Kirkness (Auckland), Nicola McLelland (Nottingham), Matthew Stevens (Oxford). 


\section{Das Phänomen Standardsprache im Englischen und im Deutschen}

In diesem Beitrag wird überwiegend ausgegangen von Begriffen und Positionen der Soziolinguistik, so wie sie in Großbritannien praktiziert wird, und bei der Vorbereitung wurden vornehmlich englischsprachige Publikationen verwendet. Es ist mir klar, dass die deutschsprachige Linguistik und deutschsprachige Werke genau so relevant sein können, ich hoffe aber, dass ein „Blick aus dem Ausland" ein neues Licht auf die Probleme werfen kann. Ich gehe insbesondere von einer Auffassung des Begriffes Standardsprache aus, die in der britischen Soziolinguistik verbreitet ist; nach diesem Verständnis des Begriffes ist ein „standard language“ der in der Gesellschaft sowohl in geschriebener als auch in gesprochener Form als maßgeblich akzeptierter Sprachgebrauch. Nach diesem Verständnis des Phänomens ist Standardsprache (ich benutze aus Bequemlichkeitsgründen den deutschen Terminus, gemeint ist aber hier durchweg eher der englische Begriff) der gesamte Sprachgebrauch einer Bildungsschicht, d.h. sie umfasst alle sprachlichen Register dieser Schicht, und schließt demnach das ein, was man im Deutschen als Umgangssprache der Bildungsschicht bezeichnen würde. Wie Trudgill (1999) klarmacht, ist diese Varietät, oder, besser gesagt, dieses Spektrum von Varietäten, leichter negativ als positiv zu bestimmen - es ist der Sprachgebrauch, der nicht allgemein als „falsch“ bezeichnet wird; unter Umständen „snobistisch" oder ,unsympathisch“, aber nicht als „falsch“. Es handelt sich hier sowohl um gesprochene als auch um geschriebene Sprache, und sowohl um formelle als auch um informelle Sprache, also auch um Umgangssprache. Standardisiert ist diese Varietät vor allem in der Grammatik und in der Orthographie, kaum in der Aussprache, und nur bedingt in der Lexik. Da es sowohl um gesprochene als auch um geschriebene Sprache geht, handelt es sich hier um einen Gebrauchsstandard oder eine Gebrauchsnorm, und nicht nur um einen kodifizierten Standard oder eine Sollnorm (s. Ammon 1995, S. 73-88); wie z. B. Cheshire (1999) überzeugend dargelegt hat, beziehen sich Sollnormen ausschließlich auf geschriebene Sprache oder auf die wenigen mündlichen Äußerungstypen, die in besonders engem Verhältnis zur Schrift stehen.

Es beziehen sich meine Bemerkungen zum Englischen fast ausschließlich auf das britische Englisch (eigentlich auf das englische Englisch); es geht mir um Probleme von Deutsch lernenden Briten, die aus unterschiedlichen Standardisierungsmustern entstehen, aber auch um Probleme deutschsprachiger Studenten, die etwa in Großbritannien ein ERASMUS-Studium absolvieren. Es geht mir auch um die englischsprachige Kommunikation zwischen Deutschen und Briten.

Um zusammenzufassen, ist das Standardenglische, so wie ich es verstehe, grammatisch standardisiert, aber mit einer breiten Palette stilistisch bedingter grammatischer Variation. Was das Lexikon betrifft, ist es, wie in jeder natürlichen Sprache, unendlich erweiterbar. Viele Lexeme, viele Wörter, die von ein- 
zelnen Englischsprachigen verstanden werden, gehören zur Zeit womöglich nicht zum Standardenglischen, sind entweder dialektal oder fremdsprachlich, könnten aber, da das Lexikon prinzipiell unbegrenzt ist, womöglich in Zukunft ins Standardenglische gelangen. Ob ein einzelnes Wort standardsprachlich ist oder nicht, ist häufig einfach Geschmackssache oder Meinungssache des Einzelnen.

Vor allem auf lexikalischer Ebene (aber auch in der Phonetik und Phonologie) sind auch die Phänomene anzutreffen, die unterschiedliche Standardvarietäten trennen, z. B. britisches Englisch, amerikanisches Englisch, usw. Auch hier ist der genaue Status von einzelnen Lexemen häufig umstritten oder kaum festzulegen; ich wüsste z. B. selber nicht ob skillet (Bratpfannelfrying pan) ausschließlich nordamerikanisch ist oder auch jetzt Teil des britischen Englisch.

Die Verständigung zwischen Sprechern unterschiedlicher Varietäten ist durch Akkomodation gewährleistet; in jeder Konversation passen sich die Gesprächspartner sprachlich an, wobei eine stärkere Anpassung von dem Gesprächspartner zu erwarten ist, dessen Varietät in der konkreten Situation weniger Wert hat. Hier spielt natürlich der geographische Ort eine Rolle, an dem konkret das Gespräch stattfindet, aber auch das Prestige der Varietäten, wobei Varietäten, die als Erste Sprachen fungieren, etwa das britische oder amerikanische Englisch, generell höheres Prestige als etwa afrikanische Varietäten genießen. Diese Prestigegefälle sind bedauerlich, aber leider eine Tatsache (s. Quirk 1985). Die gegenseitige Verständigung ist hierbei in der Regel gut - es scheint tatsächlich hier ein Gesetz der Kommunikation zu fungieren; wenn Menschen über ein Minimum an gemeinsamen sprachlichen Mitteln verfügen und wo gegenseitig guter Wille vorhanden ist, werden sie sich verständigen. Kommunikationsstörungen entstehen vor allem dann, wenn bestimmte Gruppen oder Individuen nicht wissen, dass sie sich anpassen müssen, oder nicht dazu imstande sind. ${ }^{1}$

Entgegen verbreiteten Meinungen im Ausland ist die Aussprache des britischen Englisch nicht standardisiert. Die maßgebende Bildungsschicht verwendet ein breites Spektrum von regional bedingten Aussprachen (,accents“ genannt), die sich vor allem auf phonetischer Ebene, weniger auf phonologischer oder systematischer Ebene voneinander unterscheiden (Ausspracheunterschiede zwischen Nordamerika und Großbritannien sind etwas häufiger systematischer Art). Die bekannte RP-Aussprache, die im Sprachunterricht für Anderssprachige vorherrscht, hat keinen Standardstatus innerhalb Großbritanniens und spielt im staatlichen Schulwesen keine Rolle, obwohl die Aussprache von Standardsprechern oft deutlich RP-beeinflusst ist - Aussprachen die auf phonologischer Ebene oder auf scheinbar unsystematischer Weise

${ }_{1}$ Zur Theorie der Akkomodation gibt es eine reichhaltige Literatur. Eine kurze einführende Skizze bietet Downes (1998, S. 271-272). 
stark von der RP abweichen sind meistens mit Nichtstandard oder mit Dialekten assoziiert. ${ }^{2}$

Wenn wir den Blick auf das Deutsche werfen, stelle ich fest, dass die Situation nicht unähnlich ist und sich offenbar der englischen Situation nähert. Was als „neue Standardvariation“ bezeichnet wird, ist für mich die wachsende Verwendung umgangssprachlicher Varianten in Situationen, die früher der Standardsprache vorbehalten waren. Oder, anders ausgedrückt, das Standarddeutsche umfasst jetzt Varianten, die früher als umgangssprachlich galten, oder es entsteht jetzt eine neue Art Standardsprache, die nicht nur die formelle Sprache einer Bildungsschicht darstellt, sondern auch die informelle Sprache dieser Gruppe. Mit anderen Worten ähneln sich das Deutsche und das Englische in ihren soziolinguistischen Eigenschaften zunehmend. ${ }^{3}$ Ähnlich ist auch die Tatsache, dass das Deutsche unterschiedliche nationale Varietäten kennt, etwa in Deutschland, Österreich und der Schweiz.

\section{Standardvariation im Deutschen; ihre Bedeutung für englischsprachige Lerner}

Wie wirkt sich die zunehmende soziolinguistische Ähnlichkeit zwischen den Sprachen auf die Aufgabe der Lerner aus? Wird die soziolinguistische Interferenz, die sehr anschaulich von Martin Durrell beschrieben wurde (Durrell 1999), möglicherweise abgebaut? Die Aufgabe des britischen Lerners des Deutschen müsste theoretisch leichter werden. Die Kluft zwischen der Standardsprache, die man lernen muss, und der Umgangssprache, die man hört, ein Hindernis für meine Generation, müsste weniger problematisch werden. Als Student der Germanistik musste ich selber in der Praxis herausfinden, dass Formen des 1. Konjunktivs und, besonders im süddeutschen Raum, die meisten Formen des Präteritums für den formellen, vor allem für den geschriebenen Sprachgebrauch charakteristisch sind; die Tatsache, dass manche solche Formen nicht ins ungezwungene Gespräch passen, wurde nicht gelernt, da im Prinzip keine Informationen über die Umgangssprache vermittelt wurden. Mit Hilfe von neueren Nachschlagewerken, z. B. Durrell (2003), stellt der heutige Student viel schneller und leichter fest, dass es im Deutschen ähnlich wie im Englischen eine informelle Standardsprache gibt, und wird über dieses Varietätenspektrum, früher als „Umgangssprache“ vernachlässigt, unter Umständen gut informiert. Es scheinen hier drei unterschiedliche aber zusammenhängende Änderungsprozesse nebeneinander zu gehen: ein erweiterter Gebrauch informeller oder umgangssprachlicher Formen; eine Aufwertung mancher Formen, die einen Standardstatus erhalten; eine Berücksichtigung informeller Formen in Nachschlagewerken und im Sprachunterricht.

${ }^{2}$ Eine deutliche und einsichtsreiche Schilderung der gesellschaftlichen Stellung und der Beschaffenheit des Standardenglischen bietet Milroy (1999).

3 Barbour/Stevenson (1998, S. 245-198) stellt den Versuch dar, das jetzige Variationsspektrum des Deutschen kurz gefasst zu schildern. 
Etwas anders als mit diesen grammatischen Vorgängen verhält es sich mit dem Phänomen des beliebig erweiterbaren Lexikons; auf diesem Gebiet besteht anscheinend in deutschen Nachschlagewerken ein gewisser Widerstand gegen den Sprachwandel: manche gängigen neueren Wörter erscheinen in Wörterbüchern nicht, weil sie halt als nicht standardsprachlich oder sogar als nicht deutsch empfunden werden. Ich rede hier vor allem von den Anglizismen; ich habe in der letzten Zeit in relativ neuen Wörterbüchern, sowohl gedruckt als auch elektronisch, zum Teil vergeblich nach Handy, Mobbing und Beamer gesucht. Es wird sich natürlich sofort hier die Frage stellen „Warum müssen Sie als Engländer diese Wörter nachschlagen, das sind eben englische Wörter?" Das sind aber keine englischen Wörter im Sinne von „Wörter der englischen Sprache“. Das sind deutsche Anglizismen, die nur mit erheblich anderer Bedeutung, wenn überhaupt, im Sprachgebrauch Englischsprachiger vorkommen; mir zumindest ist z. B. Beamer im Englischen völlig unbekannt. Die Unterschiede zwischen deutschen Anglizismen und Wörtern der englischen Sprache wurden häufig von englischsprachigen Germanisten betont; besonders hervorzuheben sind die Werke von Alan Kirkness', z. B. Kirkness (2001). ${ }^{4}$

\section{Standardvariation im Englischen; ihre Bedeutung für deutschsprachige Lerner}

Wie sieht es für deutsche Anglisten aus? Wichtige Quellen der soziolinguistischen Interferenz könnten hier abgebaut werden. Es wäre möglicher Weise jetzt leichter zu verstehen, gegen den Hintergrund der Lockerung von Normen im Deutschen, dass auch das Standardenglische eher flexible Normen kennt. Dies könnte sogar die Lernaufgabe der deutschsprachigen Anglisten erleichtern; z. B. könnte die Anerkennung, dass die englische RP-Aussprache keine Standardaussprache darstellt, dazu führen, dass Lerner des britischen Englisch eine andere Aussprache als RP wählen; RP ist nämlich für Deutschsprachige besonders schwierig, da die Vokalunterschiede, vor allem zwischen den betonten Vokalen der Wörter „cattle“ (d.h. Rindvieh) und „kettle“ (d.h. Kessel) für sie kaum hörbar ist. Eine westenglisch oder nordenglisch gefärbte Aussprache wäre für Deutschsprachige in dieser Hinsicht erheblich leichter, und durchaus salonfähig (aber natürlich herrschen immer häufiger angestrebte amerikanische Aussprachen vor, die aber auch für Deutschsprachige schwierige Vokalunterscheidungen aufweisen).

Eine zweite Interferenzquelle entsteht eigentlich aus der sehr guten und genauen Beschreibung des Standardenglischen, die den deutschen Unterrichts-

${ }^{4}$ Für Kirkness sind viele „englische Wörter" einfach Wörter des Deutschen, die nur scheinbar aus dem Englischen kommen. Den Terminus „Anglizismus" verwendet er lediglich für die Formen, die tatsächlich im Sprachgebrauch Englischsprachiger nachzuweisen sind. 
plänen zugrunde liegt; es wird nämlich, was zu begrïßen ist, durchaus ein "standard English" nach englischem Muster gelehrt, besonders was die Grammatik betrifft, d.h. man unterrichtet sowohl die formelle Standardsprache als auch die informelle Umgangssprache; bisher haben aber deutsche Studenten anscheinend angenommen, sie lernen eine formelle Standardsprache nach deutschem Verständnis. Da z. B. die kontrahierten Verbalformen don't, can't usw. unterrichtet werden, wird angenommen sie seien formell standardsprachlich, während sie z. B. in Aufsätzen nicht unbedingt am Platze sind.

\section{Die Aufgabe deutschsprachiger Lerner des Englischen; die Rolle des „deutschen Englisch“}

Bei der weiteren Besprechung von Problemen, die die Kommunikation in englischer Sprache zwischen Deutschsprachigen und Englischsprachigen möglicher Weise beeinträchtigen, stelle ich zunächst die These auf, dass es heutzutage ein „deutsches Englisch" gibt. Diese These entsteht vor allem aus meinen häufigen Gesprächen auf Englisch mit deutschsprachigen Studenten, die an britischen Universitäten Anglistik studieren. Mit dem Terminus „deutsches Englisch " möchte ich andeuten, dass dies jetzt eine relative stabile Varietät ist, mit zum Teil vorhersagbaren Eigenschaften; mit dem Terminus deute ich bewusst auch Parallelen zu etablierteren Varietäten wie etwa indischem oder nigerianischem Englisch an. Ein so geartetes „deutsches Englisch“ wäre eine Untervariante des europäischen Englisch. In der Tabelle führe ich einige Beispiele für Phänomene des „deutschen Englisch“ auf; ich betone ausdrücklich, dass diese Beispiele rein aus informellen, aber langjährigen, persönlichen Beobachtungen stammen, und nicht etwa aus einem soziolinguistischen Forschungsprogramm.

Bei der Besprechung des „deutschen Englisch“ bin ich leider auf solche informellen Beobachtungen angewiesen, da anscheinend noch keine wissenschaftlich fundierten Beschreibungen vorliegen, obwohl sie vorbereitet werden, z. B. von Barbara Seidlhofer (Wien). Die Titel einiger Werke geben den Eindruck, sie beschreiben ein deutsches oder europäisches Englisch, während sie in Wirklichkeit ein anderes Phänomen behandeln. Z.B. bezieht sich das Buch „English in Europe“ (Görlach 2002) nicht auf den Gebrauch des Englischen durch Deutschsprachige usw., sondern auf den vom Englischen beeinflussten Gebrauch anderer Sprachen, einschließlich des Deutschen. Es geht mir hier also nicht um ein vom Englischen beeinflusstes Deutsch („Denglisch"), das häufig behandelt wurde, und das in der Kommunikation zwischen Deutschsprachigen verwendet wird, sondern um die Varietät des Englischen, das von Deutschsprachigen in der Kommunikation mit Anderssprachigen, einschließlich mit Englischsprachigen, verwendet wird

Das von Marko Modiano geschilderte „mid-Atlantic English“ (Modiano 2002) ist anscheinend ein anderes Phänomen, eine bereits standardisierte von Europäern benutzte Kompromissvarietät zwischen britischem und amerika- 


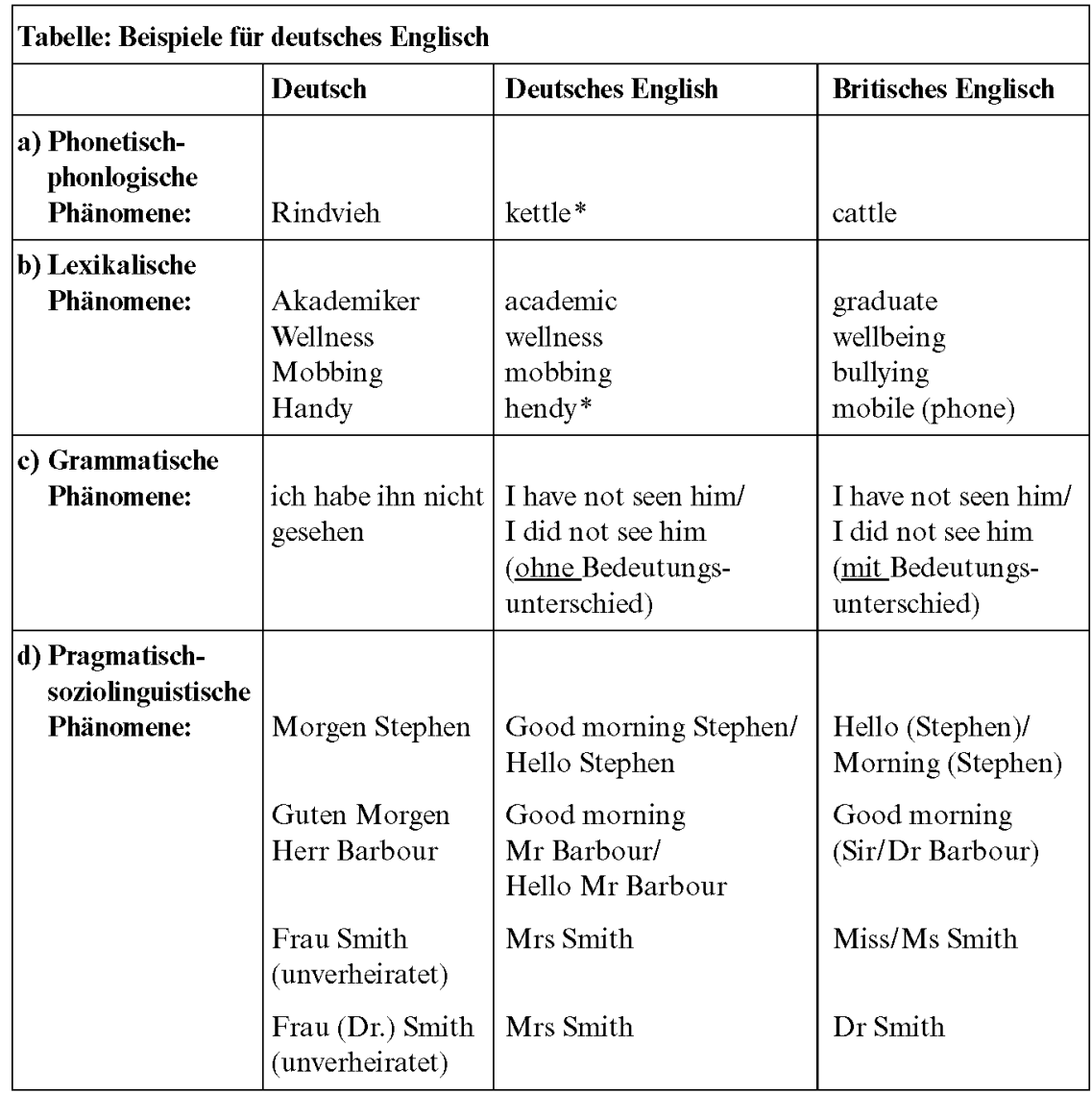

* Diese Wörter werden in einer Form angegeben, die ihre Wahrnehmung durch Englischsprachige impressionistisch wiedergibt

nischem Englisch; das von mir beschriebene deutsche Englisch ist, obwohl in seinen Eigenschaften teilweise vorhersagbar, noch nicht standardisiert, und fällt vielmehr durch Beeinflussung durch das Deutsche auf, als durch einen britisch-amerikanischen Mischcharakter.

Gegen die These eines „deutschen Englisch“ könnte argumentiert werden, es handle sich um keine stabile Varietät, sondern einfach um eine inkorrekte Lernervarietät, die der Verbesserung bedarf; indirekte Beschreibungen der Varietät kommen dementsprechend in Werken vor, die darauf abzielen, die „Fehler" im Englisch der Deutschsprachigen zu beseitigen. ${ }^{5}$ Mangels wissenschaftlich fundierter Forschungen möchte ich zur Zeit nicht definitiv zwischen den Positionen entscheiden; ich bespreche die Kommunikationsprobleme vor

\footnotetext{
5 Siehe z. B. Parkes/Cornell (1993).
} 
dem Hintergrund der Annahme eines „deutschen Englisch“, möchte aber prinzipiell die Frage offen lassen, ob es berechtigt ist, von einer solchen Varietät auszugehen, oder ob man nicht eher einfach von den „Fehlern“ im Englisch der Deutschsprachigen sprechen sollte.

Inwiefern entstehen Probleme aus dem Phänomen des „deutschen Englisch"? Problematisch ist diese Varietät, weil sie für Englischsprachige gelegentlich nur schwer verständlich ist. Diese Probleme sind zu überwinden dadurch, dass Deutschsprachige anerkennen, dass sie eine besondere Varietät des Englischen sprechen und dass sie sich im Gespräch oder in der schriftlichen Äußerung entweder an das Englisch der Englischsprachigen anpassen müssen, oder bereit sein müssen, spezifische Ausdrücke ihres „deutschen Englisch" zu erklären. Schwierig ist der Prozess, weil Deutschsprachige meistens nicht anerkennen, dass sie ein „deutsches Englisch“ sprechen; häufig wird zwar anerkannt, dass grammatische Fehler gemacht werden ${ }^{6}$, die lexikalischen Eigenschaften des „deutschen Englisch“ scheinen weitgehend unerkannt zu bleiben. Diese fehlende Anerkennung ist aus dem Entstehungsvorgang des „deutschen Englisch“" zu verstehen. Diese Varietät entsteht selbstverständlich aus dem allgemein bekannten Vorgang der Interferenz (Edwards 1994, S. 72 78); Eigenschaften des Deutschen beeinflussen, oft auf recht komplexe Weise, das Englisch der Deutschsprachigen. Besonders irritierend sind hierbei lexikalische Interferenzen; im Folgenden beschränke ich mich auf solche lexikalischen Eigenschaften des ,deutschen Englisch“.

Für deutschsprachige Anglisten entstehen paradoxer Weise die hartnäckigsten lexikalischen Interferenzen in ihrem Englisch aus Änderungen in der deutschen Sprache; in diesem Abschnitt wird also der Kreis geschlossen, indem die neue Standardvariation des Deutschen mit der Beherrschung des Englischen durch Deutschsprachige in Verbindung gebracht wird. Im Mittelpunkt der Diskussion stehen hier wieder die deutschen Anglizismen. Auf die genaue Beschaffenheit dieses Phänomens gehe ich hier nicht ein ${ }^{7}$, ich betone aber die Tatsache, dass die betreffenden Vokabeln im Volksmund „englische Wörter" genannt werden, was in hohem Maße irreführend ist.

Bei den in der Tabelle aufgeführten Beispielen für das Lexikon des „deutschen Englisch" gibt es Beispiele für unterschiedliche Vorgänge bei Beeinflussung des deutschen Englisch durch das Deutsche. Bei Akademikerlacademic geht es einfach um falsche Freunde, aber es spielt hier vielleicht die Vorstellung eine Rolle, Latinismen seien im Englischen so häufig, dass es im Englischen eine einfache Entsprechung für jedes deutsche Latinismus geben müsste. Bei wellness handelt es sich um einen Amerikanismus; in Großbritannien kommen auch Amerikanismen unentwegt in den Sprachgebrauch, dieser

${ }^{6}$ Ich teile nicht unbedingt die Auffassung, dass es hier um ein ,fehlerhaftes Englisch' handelt; man könnte durchaus der Meinung sein, es entsteht einfach eine neue Varietät des Englischen.

${ }^{7}$ S. Barbour (2002). 
aber ist nicht geläufig, und ist, zumindest für mein Sprachgefühl, kaum ein mögliches britisches Wort - es klingt grammatisch falsch. Mobbing kommt im britischen Englisch zwar vor, ist aber selten, und kann sowohl Positives als auch Negatives bedeuten. Positiv heißt es ungefähr Zujubeln, Unterstützung durch eine Menge, auf den Schultern tragen. Ein Nomen handy existiert bekannter Weise im Englischen nicht.

Wie kann man die Missverständnisse vermeiden, die durch den Gebrauch des deutschen Englisch entstehen? Man könnte die betreffenden Phänomene einfach als „falsch“ ankreiden und versuchen, sie zu beseitigen. Das ist meines Erachtens nicht nötig; das deutsche Englisch hat sich als Varietät zum Teil schon etabliert und erfüllt für Deutschsprachige bereits wichtige Funktionen. Die meisten Englischsprachigen, die keine Germanisten sind, werden das vielleicht auch nicht als „falsch“ empfinden; sie sind es bereits gewohnt, dass es ausländische Varietäten des Englischen gibt, die nicht unbedingt völlig verständlich sind; liberale Briten haben auch oft die Auffassung, man müsste den Versuch machen, fremde Varietäten des Englischen zu verstehen, und könnten sogar unter Umständen der Meinung sein, sie seien selbst zum Teil an den Missverständnissen schuld. Ein deutsches Englisch möchte ich also keineswegs beseitigen, ich möchte aber seine Sprecher darauf aufmerksam machen, das es das gibt. Ich möchte sie darauf hinweisen, dass sie sich im englischen Gespräch mit Nichtdeutschsprachigen vielleicht an die Sprache des anderen anpassen wollen, genau wie sich der Australier in England oder der Engländer in Amerika sich anpasst. Als erster Schritt zur Erklärung des deutschen Englisch, müsste man unbedingt auf die Bezeichnung „englische Wörter“ für die Anglizismen in der deutschen Sprache verzichten.

\section{Schluss}

Die neue Standardvariation im Deutschen scheint eine bisherige Schranke zwischen den Sprachen, ihr unterschiedliches soziolinguistisches Profil, womöglich abzubauen, da sie jetzt beide über relativ variable Standardvarietäten, mit einer eher dem Gebrauch orientierten Norm, verfügen. Gleichzeitig aber entsteht eine neue Schranke daraus, dass sich das Deutsche dem Englischen scheinbar nähert, aber auf eine sehr komplexe Weise, die in der Praxis, unter dem Schlagwort „englische Wörter im Deutschen“ grob vereinfacht wird.

Eine adäquate Berücksichtigung der neuen Standardvariation setzt voraus, dass man sich bereits am Anfang des Sprachunterrichts mit der gesellschaftlichen Seite der Sprache befasst - das deutsche Verhältnis zu den Anglizismen ist zum Beispiel ein Kapitel der Soziolinguistik. Allen Erscheinungen, die ich in diesem Referat behandelt habe kann man nur gerecht werden, wenn man die Sprache in ihrem Verhältnis zur Gesellschaft betrachtet. Die Thematik Sprache und Gesellschaft gehört nicht nur in ein Hauptseminar sondern auch in die Anfängerstunde. 


\section{Literatur}

Ammon, Ulrich (1995): Die deutsche Sprache in Deutschland, Österreich und der Schweiz. Berlin/New York.

Barbour, Stephen (2002): Die Verteidigung der Sprache. Noch einmal die Fremdwortfrage im Deutschen. In: Hartung, Wolfdietrich/Shethar, Alissa (2002) (Hg.): Kulturen und ihre Sprachen. Die Wahrnehmung anders Sprechender und ihr Selbstverständnis. S. $235-242$.

Barbour, Stephen/Stevenson, Patrick (1998): Variation im Deutschen. Soziolinguistische Perspektiven. Berlin/New York.

Bex, Tony/Watts, Richard J.(1999) (Hg.): Standard English. The Widening Debate. London/New York.

Cheshire, Jenny (1999): Spoken standard English. In: Bex, Tony/Watts, Richard J.(1999) (Hg.): Standard English. The Widening Debate. S. 129-148.

Davies, Máire C./Flood John L./Yeandle, David N. (2001) (Hg.): ,Proper Words in Proper Places'. Studies in Lexicology and Lexicography in Honour of William Jervis Jones. Stuttgart.

Downes, William (1998): Language and Society. Cambridge.

Durrell, Martin (1999): Standardsprache in England und Deutschland. In: Zeitschrift für germanistische Linguistik 2. S. 285-308.

Durrell, Martin (2003) (2. Auf1.): Using German: A Guide to Contemporary Usage. Cambridge

Edwards, John (1994): Multilingualism. London/New York.

Görlach, Manfred (2002) (Hg.): English in Europe. Oxford.

Hartung, Wolfdietrich/Shethar, Alissa (2002) (Hg.): Kulturen und Ihre Sprachen. Die Wahrnehmung anders Sprechender und ihr Selbstverständnis. Berlin

Kirkness, Alan (2001): Anglicisms, borrowings and pseudo-borrowings in German: -ical revisited: In: Davies, Máire C./Flood John L./Yeandle, David N. (2001) (Hg.): ,Proper Words in Proper Places'. Studies in Lexicology and Lexicography in Honour of William Jervis Jones. S. 329-333.

Linn, Andrew R./McLelland, Nicola (2002) (Hg.): Standardization. Studies from the Germanic Languages. Amsterdam/Philadelphia

Milroy, Lesley (1999): Standard English and language ideology. In: Bex, Tony/Watts, Richard J. (1999) (Hg.): Standard English. The Widening Debate. S. 173-206

Modiano, Marko (2002): Standardization Processes and the mid-Atlantic English paradigm. In: Linn, Andrew R./McLelland, Nicola (2002) (Hg.): Standardization. Studies from the Germanic Languages. S. 229-252.

Parkes, Geoff/Cornell, Alan (1993): German-English False Friends, Reference \& Practice. Vol. 3. Southampton.

Quirk, Randolph (1985) The English language in a global context. In: Quirk, Randolph/ Widdowson, Henry G. (1985) (Hg.) English in the World. S.1-6.

Trudgill, Peter (1999): Standard English; What it isn't. In: Bex, Tony/Watts, Richard J. (1999) (Hg.): Standard English. The Widening Debate. S. 117-128. 